\title{
SOME COMMENTS ON THE THEOREM PROVIDING STATIONARITY CONDITION FOR GSTAR MODELS IN THE PAPER BY BOROVKOVA et al.
}

\author{
SuHARTONO AND SUbANAR
}

\begin{abstract}
Generalized Space-Time Autoregressive (GSTAR) model is one of the models that usually used for modeling and forecasting space and time series data. The aim of this paper is to study further about the stationarity conditions for parameters in the GSTAR model and the relation to Vector Autoregressive (VAR) model. We focus on the theoretical study about stationarity condition in $\operatorname{GSTAR}\left(1_{1}\right)$ and the relation to the stationarity condition of parameters in $\operatorname{VAR}(1)$. Then, we do an empirical study to give counter examples for the theorem of stationarity condition proposed by Borovkova et al. The results show that the theorem of stationarity condition of parameters in $\operatorname{GSTAR}\left(1_{1}\right)$ model given by Borovkova et al. is incorrect. Additionally, the empirical results also show that GSTAR(1 $\left.1_{1}\right)$ model could always be represented in $\operatorname{VAR}(1)$ model by applying matrix operation to the space and time parameters. Hence, we can also conclude that VAR model, particularly $\operatorname{VAR}(1)$, is an extension of $\operatorname{GSTAR}\left(1_{1}\right)$ model with any possibility values of space and time parameters.
\end{abstract}

\section{INTRODUCTION}

The time series data in many empirical studies consist of observations from several variables, known as multivariate time series data [2]. For example, in a sales performance study, the variables might include sales volume, prices, sales force, and advertising expenditures.

In daily life, we frequently deal with the data that depend not only on time (with past observations) but also on site or space, called spatial data. Space-time

Received 10 March 2006, revised 14 September 2006, accepted 4 October 2006

2000 Mathematics Subject Classification: 62M45

Key words and Phrases: stationarity condition, GSTAR, VARIMA, space and time series 
model is a model that combines time and space dependence which affects certain multivariate time series data. This model firstly proposed by Pfeifer and Deutsch (see $[7,8])$.

In this paper, we will do a theoretical study about stationary condition of parameters in GSTAR models related to a VAR model. Then, we will also give numerical illustrations in many possibilities of parameter values in GSTAR model. Some previous comparison studies particularly on the comparison of building model steps between GSTAR and VARIMA models can be found in [9].

\section{VECTOR AUTOREGRESSIVE (VAR) MODEL}

Let $z_{i}(t)$, where $t \in T, T=\{1,2, \ldots, T\}$ is time parameter index and $i=\{1,2, \ldots, N\}$ is counted and limited variable (as an example is monthly oil production at different locations), then $\operatorname{VAR}(\mathrm{p})$ model in general is defined as (see $[3,4,10])$

$$
Z(t)=\Phi_{1} Z(t-1)+\Phi_{2} Z(t-2)+\ldots+\Phi_{p} Z(t-p)+e(t)
$$

where $Z(t)$ is vector of multivariate time series that is corrected by mean, $\Phi_{p}(B)$ is the autoregressive matrix polynomial of order $i$, and $e(t)$ is a white noise errors vector.

Stationarity condition of $\operatorname{VAR}(\mathrm{p})$ model could be found in many time series books, such as in $[3,4,10]$. The proposition about stationarity condition of $\operatorname{VAR}(\mathrm{p})$ model is as follows:

Proposition 2.1. The $\operatorname{VAR}(p)$ model is stationary process if all the eigenvalues of the autoregressive matrix polynomial order $p$ that satisfy

$$
\left|I_{n} \lambda^{p}-\Phi_{1} \lambda^{p-1}-\Phi_{2} \lambda^{p-2}-\ldots-\Phi_{p}\right|=0
$$

are inside the unit circle, or $|\lambda|<1$.

Proof. See [4], page 285-286.

For illustration, the $\operatorname{VAR}(1)$ model for three variables is given by

$$
Z(t)=\Phi_{1} Z(t-1)+e(t)
$$

or

$$
\left(\begin{array}{l}
z_{1}(t) \\
z_{2}(t) \\
z_{3}(t)
\end{array}\right)=\left(\begin{array}{lll}
\phi_{11} & \phi_{12} & \phi_{13} \\
\phi_{21} & \phi_{22} & \phi_{23} \\
\phi_{31} & \phi_{32} & \phi_{33}
\end{array}\right)\left(\begin{array}{l}
z_{1}(t-1) \\
z_{2}(t-1) \\
z_{3}(t-1)
\end{array}\right)+\left(\begin{array}{l}
e_{1}(t) \\
e_{2}(t) \\
e_{3}(t)
\end{array}\right)
$$

This VAR(1) model is a stationary process if it satisfies stationarity condition in 
Proposition 2.1., that is all the eigenvalues satisfying $\left|I_{3} \lambda-\Phi_{1}\right|=0$ lie inside the unit circle or $|\lambda|<1$.

\section{GENERALIZED SPACE-TIME AUTOREGRESSIVE (GSTAR) MODEL}

GSTAR model is an extension of STAR model and has more flexibility in modeling. Space-Time Autoregressive Model order $p_{1}$ (that means the spatial order is 1 and time order is $p)$ or $\operatorname{STAR}\left(p_{1}\right)$ introduced by Pfeifer and Deutsch [4] defined as follows:

$$
Z(t)=\sum_{k=1}^{p}\left[\phi_{k 0} W^{(0)} Z(t-k)+\phi_{k 1} W^{(1)} Z(t-k)\right]+e(t)
$$

where

- $\phi_{k l}$ : is the autoregressive and the space time parameter at time lag $k$ and spatial lag $l$,

- $W^{(l)}:(\mathrm{N} \times \mathrm{N})$ the weight matrix for spatial lag $l$ (where $\left.l=0,1\right)$,

- $e(t)$ : the $\mathrm{N}$ variate white noise vector with mean 0 and variance-covariance $\sigma^{2} I_{N}$

- $Z(t)$ : $(\mathrm{N} \times 1)$ random vector at time $t$, or $Z(t)=\left[z_{1}(t) \cdots z_{N}(t)\right]^{\prime}$.

The $\operatorname{GSTAR}\left(p_{1}\right)$ model has mathematically the same notation with $\operatorname{STAR}\left(p_{1}\right)$ at equation (4). The main difference between both models are the possible values of model parameters. In $\operatorname{STAR}\left(p_{1}\right)$ model, the autoregressive and space-time parameters $\left(\phi_{k l}\right)$ are assumed to be the same for all locations. On the contrary, the $\operatorname{GSTAR}\left(p_{1}\right)$ model allows these parameters to vary with locations and it's more realistic in practice. The $\operatorname{GSTAR}\left(p_{1}\right)$ model is defined as (see $\left.[1,6]\right)$

$$
Z(t)=\sum_{k=1}^{p}\left[\Phi_{k 0}+\Phi_{k 1} W\right] Z(t-k)+e(t)
$$

where

- $\Phi_{k 0}=\operatorname{diag}\left(\phi_{k 0}^{1}, \ldots, \phi_{k 0}^{N}\right)$ and $\Phi_{k 1}=\operatorname{diag}\left(\phi_{k 1}^{1}, \ldots, \phi_{k 1}^{N}\right)$,

- weights are selected to satisfy $w_{i i}=0$ and $\sum_{i \neq j} w_{i j}=1$.

As an example, the $\operatorname{GSTAR}\left(1_{1}\right)$ model for monthly oil production case in three different location can be written as

$$
Z(t)=\left[\Phi_{10}+\Phi_{11} W\right] Z(t-1)+e(t)
$$


or

$$
\begin{aligned}
\left(\begin{array}{l}
z_{1}(t) \\
z_{2}(t) \\
z_{3}(t)
\end{array}\right)= & \left(\begin{array}{ccc}
\phi_{10} & 0 & 0 \\
0 & \phi_{20} & 0 \\
0 & 0 & \phi_{30}
\end{array}\right)\left(\begin{array}{l}
z_{1}(t-1) \\
z_{2}(t-1) \\
z_{3}(t-1)
\end{array}\right)+ \\
& \left(\begin{array}{ccc}
\phi_{11} & 0 & 0 \\
0 & \phi_{21} & 0 \\
0 & 0 & \phi_{31}
\end{array}\right)\left(\begin{array}{ccc}
0 & w_{12} & w_{13} \\
w_{21} & 0 & w_{23} \\
w_{31} & w_{32} & 0
\end{array}\right)\left(\begin{array}{l}
z_{1}(t-1) \\
z_{2}(t-1) \\
z_{3}(t-1)
\end{array}\right)+\left(\begin{array}{l}
e_{1}(t) \\
e_{2}(t) \\
e_{3}(t)
\end{array}\right)
\end{aligned}
$$

Parameter estimation of the GSTAR model can be done by applying least squares method. The theory and methodology about GSTAR model can be read extensively in [1] and [6].

\section{STATIONARITY CONDITIONS FOR PARAMETERS IN GSTAR MODEL AND THE RELATION TO VAR MODEL}

Borovkova et al. [1] and Ruchjana [6] stated that the GSTAR model, particularly GSTAR $\left(1_{1}\right)$ model, is a restricted version of the VAR model. Hence, the stationarity condition of GSTAR model can be derived from the stationarity condition of VAR model. The investigation about stationarity condition of VAR model can be seen at [5].

In particular, GSTAR $\left(1_{1}\right)$ model, $Z(t)=\left[\Phi_{10}+\Phi_{11} W\right] Z(t-1)+e(t)$ can be represented as $\operatorname{VAR}(1)$ model, $Z(t)=\Phi_{1} Z(t-1)+e(t)$, where

$$
\Phi_{1}=\left[\Phi_{10}+\Phi_{11} W\right] .
$$

Thus, GSTAR model in general is a stationary process if all the eigenvalues of matrix $\left[\Phi_{10}+\Phi_{11} W\right]$ satisfy Proposition 1, i.e. all the eigenvalues lie inside the unit circle or $|\lambda|<1$.

Borovkova et al. [1] and Ruchjana [6] applied Proposition 2.1. and yielded a theorem giving stationarity condition for $\operatorname{GSTAR}\left(1_{1}\right)$ model, i.e.

Theorem 4.1. The GSTAR $\left(1_{1}\right)$ model is a stationary process, if all parameters $\phi_{i 0}$ in $\Phi_{10}$ and $\phi_{i 1}$ in $\Phi_{11}$ satisfy

$$
\left|\Phi_{i 0}+\Phi_{i 1}\right| \leq 1 \quad \text { and } \quad\left|\Phi_{i 0}-\Phi_{i 1}\right| \leq 1 .
$$




\section{COUNTER EXAMPLES FOR STATIONARITY CONDITIONS FOR GSTAR $\left(1_{1}\right)$ MODEL GIVEN IN THE PAPER BY BOROVKOVA et al.}

This section will show empirically some cases where stationarity condition in the theorem by Borovkova et al. is not correct and inappropriate when Proposition 2.1. is used as basis of the derivation a theorem.

\section{$\diamond$ Case 1 .}

The condition when there is one or more parameters at $\Phi_{10}$ have value 1 , and all parameters at $\Phi_{11}$ are 0 . As an example, assume that monthly oil production at three locations follow $\operatorname{GSTAR}\left(1_{1}\right)$ model as

$$
\begin{aligned}
\left(\begin{array}{l}
z_{1}(t) \\
z_{2}(t) \\
z_{3}(t)
\end{array}\right)= & \left(\begin{array}{lll}
1 & 0 & 0 \\
0 & 1 & 0 \\
0 & 0 & 1
\end{array}\right)\left(\begin{array}{c}
z_{1}(t-1) \\
z_{2}(t-1) \\
z_{3}(t-1)
\end{array}\right)+ \\
& \left(\begin{array}{lll}
0 & 0 & 0 \\
0 & 0 & 0 \\
0 & 0 & 0
\end{array}\right)\left(\begin{array}{ccc}
0 & w_{12} & w_{13} \\
w_{21} & 0 & w_{23} \\
w_{31} & w_{32} & 0
\end{array}\right)\left(\begin{array}{l}
z_{1}(t-1) \\
z_{2}(t-1) \\
z_{3}(t-1)
\end{array}\right)+\left(\begin{array}{l}
e_{1}(t) \\
e_{2}(t) \\
e_{3}(t)
\end{array}\right)
\end{aligned}
$$

Based on the Theorem 1 proposed by Borovkova et al., this model is concluded as a stationary process.

This conclusion is contradictory with the results of stationarity condition of VAR(1) model based on Proposition 2.1. All the eigenvalues of model (9) have value 1 and it means that stationarity condition, i.e. all the eigenvalues inside unit circle or $|\lambda|<1$, is not satisfied.

\section{$\diamond$ Case 2.}

The condition when there is one or more parameters $\phi_{i 0}$ and $\phi_{i 1}$ have the additional results of more than 1 . As an example, assume that monthly oil production at three locations have the same distance, so we can use uniform weight location, follow GSTAR $\left(1_{1}\right)$ model as

$$
\begin{aligned}
\left(\begin{array}{l}
z_{1}(t) \\
z_{2}(t) \\
z_{3}(t)
\end{array}\right) & =\left(\begin{array}{ccc}
0.3 & 0 & 0 \\
0 & 0.5 & 0 \\
0 & 0 & 0.3
\end{array}\right)\left(\begin{array}{l}
z_{1}(t-1) \\
z_{2}(t-1) \\
z_{3}(t-1)
\end{array}\right)+ \\
& \left(\begin{array}{ccc}
0.4 & 0 & 0 \\
0 & 0.6 & 0 \\
0 & 0 & 0.8
\end{array}\right)\left(\begin{array}{ccc}
0 & 0.5 & 0.5 \\
0.5 & 0 & 0.5 \\
0.5 & 0.5 & 0
\end{array}\right)\left(\begin{array}{l}
z_{1}(t-1) \\
z_{2}(t-1) \\
z_{3}(t-1)
\end{array}\right)+\left(\begin{array}{l}
e_{1}(t) \\
e_{2}(t) \\
e_{3}(t)
\end{array}\right)
\end{aligned}
$$

By applying Theorem 4.1., we conclude that model (10) is not a stationary process because at the locations 2 and 3 given

$$
\left|\phi_{20}+\phi_{21}\right|=|0.5+0.6|=1.1 \quad \text { and } \quad\left|\phi_{30}+\phi_{31}\right|=|0.3+0.8|=1.1 .
$$


The result does not satisfy stationarity condition of $\operatorname{GSTAR}\left(1_{1}\right)$ model based on the Theorem 4.1. that require $\left|\phi_{i 0}+\phi_{i 1}\right| \leq 1$

This conclusion is also contradictory with the result of stationarity condition by using $\operatorname{VAR}(1)$ representation. Model (10) can be written as VAR(1) model, i.e.

$$
\left(\begin{array}{l}
z_{1}(t) \\
z_{2}(t) \\
z_{3}(t)
\end{array}\right)=\left(\begin{array}{lll}
0.3 & 0.2 & 0.2 \\
0.3 & 0.5 & 0.3 \\
0.4 & 0.4 & 0.3
\end{array}\right)\left(\begin{array}{c}
z_{1}(t-1) \\
z_{2}(t-1) \\
z_{3}(t-1)
\end{array}\right)+\left(\begin{array}{c}
e_{1}(t) \\
e_{2}(t) \\
e_{3}(t)
\end{array}\right) .
$$

By using matrix operation, the eigenvalues of this autoregressive matrix are $\lambda_{1}=$ $0.964, \lambda_{2}=0.143$ and $\lambda_{3}=-0.007$ respectively. All these eigenvalues satisfy the stationarity condition at Proposition 2.1.. Hence, on the VAR(1) representation, this model is a stationary process.

Based on the two previous empirical results, we can conclude that Theorem 4.1. proposed by Borovkova et al. is incorrect. This error may be caused by the derivation process to get Theorem 4.1. yielding an error when location weights or $w_{i j}$ are eliminated. Thus, we can apply Proposition 2.1. based on the VAR(1) model representation for examination of stationarity condition in $\operatorname{GSTAR}\left(1_{1}\right)$ model.

\section{CONCLUSION}

There are two main conclusions that could be made based on the previous results, i.e.

1. Stationarity condition of parameters at GSTAR $\left(1_{1}\right)$ model proposed by Borovkova et al. [1] as stated in Theorem 4.1. is incorrect.

2. Examination of stationarity condition of GSTAR model, particularly the parameters at GSTAR $\left(1_{1}\right)$ model, is correct if done by using Proprosition 2.1. with the $\operatorname{VAR}(1)$ model representation. Hence, $\operatorname{GSTAR}\left(1_{1}\right)$ model should be firstly represented as $\operatorname{VAR}(1)$ model to check stationarity condition of the parameters.

Additionally, further research on an extension of GSTAR models particularly on the flexibility of the space time parameters and on the seasonal problem is needed.

Acknowledgement. The authors would like to thank the referee for his valuable comments. 


\section{REFERENCES}

1. S.A. Borovkova, H.P. LopuhaA and B.N. Ruchjana, "Generalized STAR model with experimental weights. In M Stasinopoulos and G Touloumi (Eds.)", Proceedings of the 17th International Workshop on Statistical Modeling, Chania-Greece (2002), 139147.

2. G.E.P. Box, G.M. Jenkins And G.C. Reinsel, Time Series Analysis: Forecasting and Control, $3^{\text {rd }}$ edition, Englewood Cliffs: Prentice Hall, 1994.

3. P.J. Brockwell And R.A. Davis, Time Series: Theory and Methods, $2^{\text {nd }}$ edition, Springer-Verlag, New York, 1991.

4. J.D. Hamilton, Time Series Analysis, New Jersey: Princeton University Press, 1994.

5. E.J. Hannan, Multiple Time Series, John Wiley and Sons, New York, 1970.

6. B.N. Ruchuana, "Curve Modeling of Oil Production by Using Generalized S-TAR Model", Forum Statistika dan Komputasi, Special Edition, IPB, Bogor, 2002

7. P.E. Pfeifer And S.J. Deutsch, "A Three Stage Iterative Procedure for Space-Time Modeling", Technometrics, 22 (1) (1980a), 35-47.

8. P.E. Pfeifer AND S.J. Deutsch, "Identification and Interpretation of First Order Space-Time ARMA Models", Technometrics, 22 (1) (1980b), 397-408.

9. Suhartono and R.M. Atok, "Comparison between VARIMA and GSTAR Model for Forecasting Space and Time Series Data", Proceeding of National Statistics Seminar VII, ITS, Surabaya, Indonesia, 2005

10. W.W.S. WeI, Time Series Analysis: Univariate and Multivariate Methods, AddisonWesley Publishing Co., USA, 1990.

Suhartono: Department of Mathematics, Gadjah Mada University, Yogyakarta 55281, Indonesia.

Permanent address: Department of Statistics, Institut Teknologi Sepuluh Nopember, Kampus ITS, Keputih, Surabaya 60111, Indonesia.

E-mail: suhartono@statistika.its.ac.id

SubanaR: Department of Mathematics, Gadjah Mada University, Yogyakarta 55281, Indonesia.

E-mail: subanar@yahoo.com 